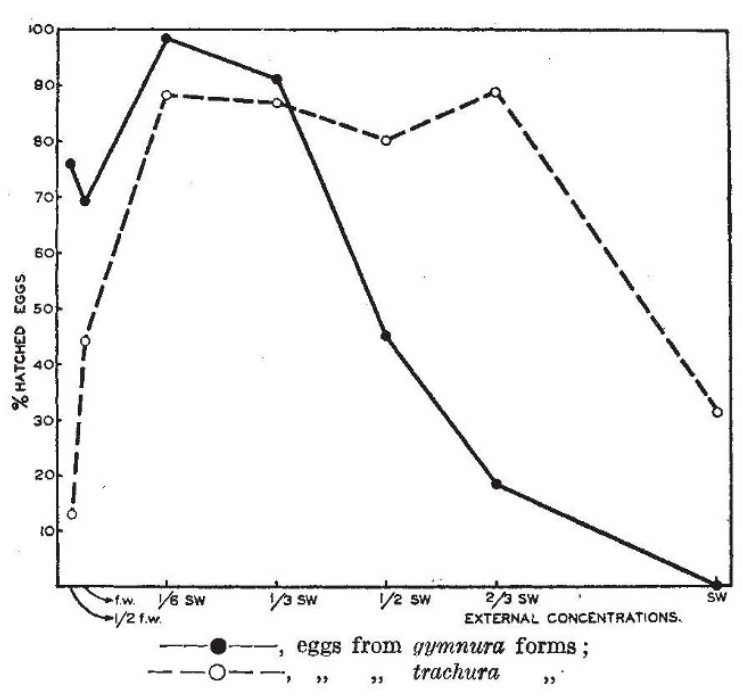

tween the number of plates and vertebræ of the parents, the percentage of viable embryos produced and their morphological characters when reared.

The geographical variation of the species as regards the number of lateral shields is also caused by an underlying genetic pattern, as will be shown by detailed publication elsewhere.

The evidence indicates that the mechanisms thus far detected, namely, the physiological differences between adults, the inherited physiological differences between their eggs, and the selective action of external factors, are powerful barriers to the diffusion of genes throughout the species, and maintain the variation at the evolutionary level actually reached.

Zoophysiological Laboratory,

M. J. Heuts

University of Louvain.

Oct. 31.

${ }^{1}$ Koch, H. J., and Heuts, M. J., Arch. Internat. Physiol., 53, 253 (1943).

' Bertin, Ann. Inst. Oceanogr., N.S., 2, Fasc. 1 (1925).

'Heuts, M. J., Ann. Soc. Roy. Zool. Belg., 76, 88 (1945).

\section{Mechanism of Crossing-over}

A NUMBER of theories have emerged from time to time attempting to visualize the exact mechanism involved during erossing-over, which is now known to be the sole agent conditioning the formation of the cytologically visible chiasma. Among these the one proposed by Darlington ${ }^{1}$ based on torsions is the most elaborate, in the sense that it offers an explanation for the long and intricate succession of events. His hypothesis, however, implies a few assumptions for which there are formidable physical objections. The most important of these are : (1) the existence of a pairing force which brings homologues together in the zygotene of meiosis, and (2) the postulate that a break at a particular level in one chromatid under strain induces a break in a non-identical chromatid at precisely the same spot due to transfer of stress. In regard to (1), every cytologist is aware of the physical difficulties which are inherent in suggesting the existence of specific attractions operating over anything more than extramolecular ranges. However, Delbrück's ${ }^{2}$ theory of autocatalytic synthesis of polypeptides appears to provide a physico-chemical basis for this force of attraction. In order to test the validity of the second assumption, an attempt is now made to examine the forces operating in a helical system which is in equilibrium under torsion.

In a two-strand rope which is held in the form of a helix, the axial force which will keep it in equilibrium in this form is

$$
\begin{aligned}
F=\frac{1}{l r^{2}}\left[\left(C \cos ^{2} \alpha+B \sin ^{2} \alpha\right)\right. & \eta+ \\
& (C-B) \sin \alpha \cos \alpha . \theta],
\end{aligned}
$$

where $l$ is length of rope; $r, \alpha$ are the radius and angle of helix ; $\eta$ is axial displacement ; $\theta$ is angular displacement at end of rope ; $C, B$ are constants. The tension at any point of the rope is constant and is $\mathcal{J}=F \sin \alpha$. $\eta$ and $\theta$ are proportional to $l$, so that at any intermediate point $Q$ of the rope, distant $l_{1}$ from the fixed end, their values are

$$
\eta_{1}=\frac{l_{1}}{l} \eta, \theta=\frac{l_{1}}{l} \theta \text {. }
$$

Hence we may also write

$$
\begin{aligned}
F=\frac{1}{l_{1} r^{2}}\left[\left(C \cos ^{2} \alpha+B \sin ^{2} \alpha\right) \eta_{1}+\right. \\
\left.(C-B) \sin \alpha \cos \alpha \cdot \theta_{1}\right] .
\end{aligned}
$$

Suppose now the string is in equilibrium under the action of $F$ applied at its end. If one of the strands is cut at $Q$, the torsional couple at that point is annulled and the two ends will revolve in opposite directions. But it is an essential feature of the theory that this release of torsional strain is confined to a narrow region near the cut. This is based on the assumption of an affinity or lateral cohesion between the two strands. Hence we may suppose that the effect of the cut is to make the loose ends revolve through small angles. Since the torsional equilibrium of the rope is due mainly to the co-existence of torsional couples in both the strands, it follows that the local release of twist in one strand will result in a corresponding release of twist in the other also at the same point. This will result in changing $\eta_{1}$ and $\theta_{1}$ at that point in the uncut strand to new values $\eta_{1}{ }^{\prime}$ and $\theta_{1}{ }^{\prime}$, which will be less than the original values $\eta_{1}$ and $\theta_{1}$. The corresponding equilibrium value of $F$, namely, $F^{\prime}$, is given by

$$
\begin{aligned}
& F^{\prime}=\frac{1}{l_{1} r^{2}}\left[\left(C \cos ^{2} \alpha+B \sin ^{2} \alpha\right) \eta_{1}{ }^{\prime}+\right. \\
& \left.(C-B) \sin \alpha \cos \alpha \cdot \theta_{1}{ }^{\prime}\right],
\end{aligned}
$$

so that

$$
F^{\prime}<E
$$

If we suppose that the rope was in limiting equilibrium under $F$, then it is clear that after one of the strands is cut, it is under the action of $F^{\prime}$ which is more than the value $F^{\prime}$ required for equilibrium. Hence there is a high probability that the uncut strand will also break at the same point, since the tension at that section is greater than the value required for equilibrium.

Thanks are due to Mr. V. R. T. Achar and to Dr. B. R. Seshachar for helpful criticism.

\section{University of Mysore, Central College, Bangalore.}

1 Darlington, C. D., J. Genet., 31, 185 (1935).

2 Delbrück, M., Cold Spring Harbor Symposia, 9, 122 (1941).

\section{K. V. SRINATH}

